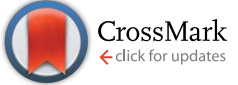

Cite this: Chem. Sci., 2015, 6, 2806

\title{
Chemical assay-guided natural product isolation via solid-supported chemodosimetric fluorescent probe $\uparrow$
}

\author{
Hongjun Jeon, Chaemin Lim, Ji Min Lee and Sanghee Kim* \\ As a new model of chemical assay-guided natural product isolation, an effective chemodosimetric assay \\ system was devised. Our chemical assay system features a fluorogenic chemodosimeter immobilized on \\ a solid support, which offers advantages in identifying the desired compounds in complex natural \\ product mixtures. To isolate only compounds with the target functional groups, the click chemistry \\ concept was adopted. The model system presented here was developed for natural products with a \\ terminal alkyne. Using our newly designed alkyne sensing beads with the aforementioned features, we \\ have readily identified, quantified, and isolated compounds with a terminal alkyne group from plant extracts.
}

Received 30th January 2015

Accepted 25th March 2015

DOI: $10.1039 / \mathrm{c} 5 \mathrm{sc00360a}$

www.rsc.org/chemicalscience

The "click reaction" is thermodynamically favorable and

\section{Introduction}

Despite the advent of modern drug discovery technologies, natural products still remain a significant source of new drugs. ${ }^{1}$ In addition, they are valuable tools with which to study biology. ${ }^{2}$ At present, bioassay-guided isolation is the favored procedure for identifying new natural substances. ${ }^{3}$ Although modern analytical and bioassay techniques have made it considerably competitive ${ }^{4}$ bioassay-guided isolation is a time-consuming process and it still has some limitations caused by false assay signals arising from compounds with unspecific activities. ${ }^{5}$ Thus, alternative and complementary approaches are needed to further advance natural product research.

The isolation of natural products can also be guided by chemical cues associated with functional groups. ${ }^{6}$ The chemical reactions of certain functional groups can produce a detectable signal and this has long been used in the natural product isolation process. ${ }^{7}$ For example, the phenolic group of tannins was identified by $\mathrm{FeCl}_{3}$, and the tertiary amino group of alkaloids was detected by Dragendorff's reagent. However, this approach has become obsolete and is not considered useful, primarily because of the low sensitivity and specificity of the chemical cues produced in natural product extracts. If an effective chemical assay system were to be developed that could easily and reliably identify a compound with a specific functional group in an extract, the systematic exploration of the natural product chemical space would be greatly facilitated.

College of Pharmacy, Seoul National University, 1 Gwanak-ro, Gwanak-gu, Seoul 151-742, Korea. E-mail: pennkim@snu.ac.kr; Fax: +82-2-888-0649; Tel: +82-2-8802487

$\uparrow$ Electronic supplementary information (ESI) available. See DOI: $10.1039 / \mathrm{c} 5 \mathrm{sc} 00360 \mathrm{a}$ reliably leads to a single product under mild reaction conditions. ${ }^{\mathbf{2}}$ We envisaged that the proper application of click chemistry would guarantee the specificity and reliability of a chemical assay system. Among various click reactions, the copper(I)-catalyzed azide-alkyne cycloaddition (CuAAC) reaction is the most popular and well-studied. ${ }^{9}$ It produces a 1,4substituted triazole from an azide and a terminal alkyne in generally excellent yield. With this reaction, we designed a chemical assay system to assess the potential of click-based chemical assay-guided natural product isolation. Herein, we present a prototype system designed for terminal alkyne-containing substances, which exhibit a wide array of biological properties. $^{\mathbf{1 0}}$

\section{Results and discussion}

As shown in Fig. 1, the designed alkyne sensing chemical assay system consists of a fluorescent chemodosimetric probe, a linker, and a solid support. The fluorescent chemodosimetric probe $^{\mathbf{1 1}}$ is the key moiety of our system; it captures terminal alkyne groups through the CuAAC reaction and produces a fluorescence signal. Immobilization of the fluorescent probe on a solid support was adopted for easy handling and tracking. Consequently, the presence of compounds with a terminal alkyne group in the analyte would lead to fluorescent responses from the solid bead. In addition, via a cleavable linker, our sensing system allows the captured target component to be released from the bead as a CuAAC product for subsequent spectroscopic analysis.

The proper choice of solid support and linker is critical to the success of any solid-phase chemistry. TentaGel ${ }^{\mathrm{TM}} \mathrm{MB}$ amino resin was chosen as a solid support because of its high swelling capacity in various solvents and its relatively large particle size. 


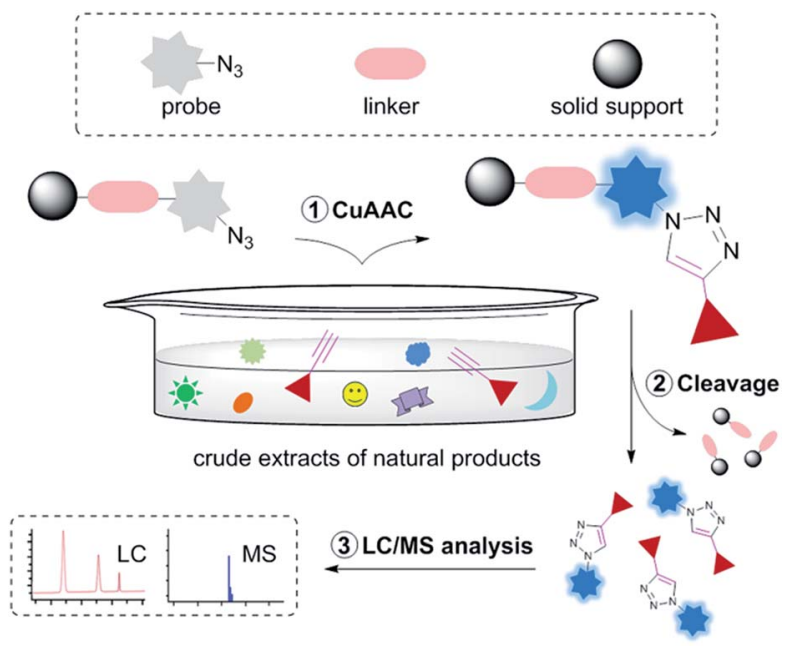

Fig. 1 A solid-supported fluorogenic chemical assay strategy for the identification of terminal alkynes in natural product extracts.

The linker chosen was (2-phenyl-2-trimethylsilyl)ethyl(PTMSEL)-linker (Fig. 2a), which can be readily cleaved by fluoride ion under mild conditions. ${ }^{12}$ The profluorophore 3 azidocoumarin was chosen as a fluorescent chemodosimetric probe because it is transformed into a highly fluorescent triazolylcoumarin after the CuAAC reaction with terminal alkynes. ${ }^{13}$ The probe was indirectly attached to the PTMSEL linker by binding through glycolic acid, allowing facile analysis by reverse-phase LC/MS after cleavage from the linker.

The alkyne sensing bead $\mathbf{1}$ was prepared with a loading level of $0.3 \mathrm{mmol} \mathrm{g}^{-1}$ using standard solid-phase synthesis techniques (see ESI $\dagger$ ). With the alkyne sensing bead $\mathbf{1}$ in hand, we explored the optimal conditions for the CuAAC reaction. 1Pentyne $(0.9 \mu \mathrm{mol})$ was used as a model alkyne substrate. After investigating various sets of conditions, we observed that treatment of bead $1(0.3 \mu \mathrm{mol}, 1 \mathrm{mg})$ with $\mathrm{CuSO}_{4} \cdot 5 \mathrm{H}_{2} \mathrm{O}(1.5$ $\mu \mathrm{mol})$, ascorbic acid $(7.5 \mu \mathrm{mol}), N, N$-diisopropylethylamine $(15.0 \mu \mathrm{mol})$, acetic acid $(30.0 \mu \mathrm{mol})$, and tris[(1-benzyl-1H-1,2,3triazol-4-yl)methyl]amine $(3.0 \mu \mathrm{mol})$ in DMF $(c a .50 \mu \mathrm{L})$ at room temperature generated the desired triazolylcoumarin product in nearly quantitative yield ${ }^{14}$ without undesirable side reactions. ${ }^{15,16}$ When 1-pentyne was reacted with bead 1 under the aforementioned conditions, fluorescence emission was observed from the beads under UV light. However, in a control experiment without the terminal alkyne, no fluorescence signal was observed. The fluorescence of these "reporter beads" was clearly distinguished by conventional fluorescence microscopy (Fig. 2b).

The resulting on-resin fluorescent product was cleaved from the solid support by treatment with $\mathrm{TBAF} \cdot 3 \mathrm{H}_{2} \mathrm{O}$ and subsequently analyzed by LC/MS. Because the detached compound had a UV-active functionality and a hydrophilic carboxylic acid group, as exemplified by compound 3 (Fig. 2e), the analysis was readily achieved using a conventional LC/MS system equipped with a diode-array UV detector and a reverse-phase column. The LC chromatogram of the analyte released from the fluorescent beads (analyte B) showed only one peak, which was identified as
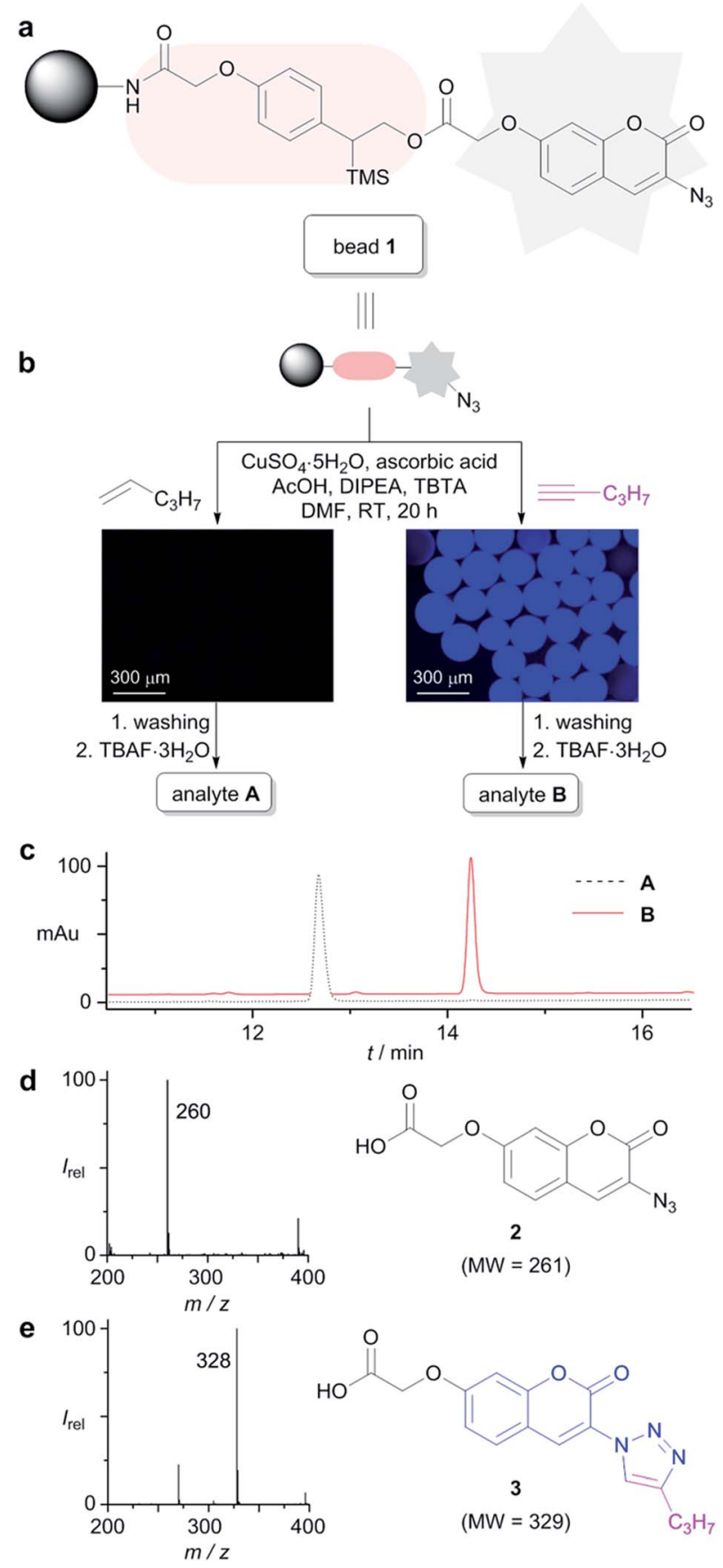

Fig. 2 Solid-supported alkyne sensing system. (a) The chemical structure of alkyne sensing bead 1; (b) schematic representation of the reaction procedure and fluorescence images of the beads (fluorescence images were obtained using excitation/emission filters of 387 $\mathrm{nm} / 434 \pm 9 \mathrm{~nm}$ and a $10 \times$ objective lens); (c) overlaid LC chromatograms of analytes A and B (at $345 \mathrm{~nm}$ ); (d) ESI negative ionization mode mass spectrum of the peaks at 12.7 min for analyte $A$ and the structure of 2; (e) ESI negative ionization mode mass spectrum of the peaks at $14.2 \mathrm{~min}$ for analyte $\mathrm{B}$ and the structure of 3 . DIPEA $=N, N-$ diisopropylethylamine; TBTA = tris[(1-benzyl-1H-1,2,3-triazol-4-yl) methyllamine; $\mathrm{MW}=$ molecular weight.

the molecular ion $[\mathrm{M}-\mathrm{H}]^{-}$at $m / z 328$ in the ESI negative ionization mode. The molecular mass (329 Daltons) of this click product matched well with the theoretical value for 
triazolylcoumarin 3. The LC chromatogram for the control experiment (analyte A) also showed only one peak with a molecular ion at $\mathrm{m} / z 260\left([\mathrm{M}-\mathrm{H}]^{-}\right)$, which matched well with the theoretical molecular weight of unreacted 3-azidocoumarin 2 (Fig. 2d).

To further validate the system, a mixture of different terminal alkynes was assayed. A mixture of equimolar amounts of four terminal alkynes $\mathbf{4 a}-\mathbf{d}$ ( 0.2 equiv. each with a maximum loading level of beads) was treated with bead 1 (Fig. 3a). The resulting fluorescent beads were treated with $\mathrm{TBAF} \cdot 3 \mathrm{H}_{2} \mathrm{O}$, and the mixture of detached compounds was analyzed by LC/MS. As shown in Fig. 3b, five peaks were observed in the LC/MS chromatogram. The molecular masses of the peaks exactly matched the theoretical values of triazolylcoumarins 5a-d and 3-azidocoumarin 2. Furthermore, the integration values of the five peaks were similar, corresponding to the theoretical ratio. The chemical yields of each compound, estimated from the integration of peak areas, were nearly quantitative. ${ }^{\mathbf{1 4}}$ This result revealed that the relative quantification of terminal alkynes is possible with this sensing system (see ESI $\dagger$ ).

To demonstrate the utility of alkyne sensing bead $\mathbf{1}$ in the natural product isolation process, it was first used in the analysis of the methanol extract of the leaves of Litsea japonica (Lauraceae). A literature search revealed that four terminal alkyne compounds 6a-d have been isolated from the leaves of $L$. japonica by two independent groups (Fig. 4a). ${ }^{17}$ As expected, the leaf methanol extract ( $3 \mathrm{mg}$ ) exhibited fluorescence activation after the click reaction with our alkyne sensing bead 1 (0.5 mg). In the LC/MS analysis of the off-bead mixture, five peaks were
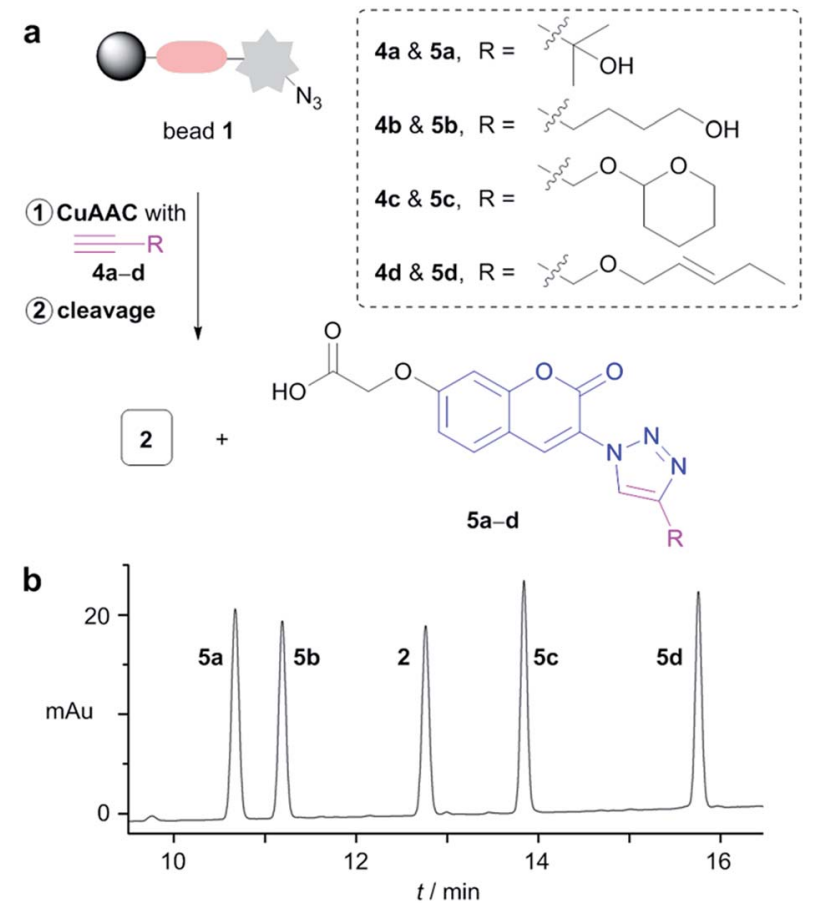

Fig. 3 Validation of the system with a set of terminal alkynes. (a) Schematic representation of the chemical assay with bead 1 and terminal alkynes $4 a-d$; (b) the LC chromatogram of the sample detached from the bead (at $345 \mathrm{~nm}$ ). a

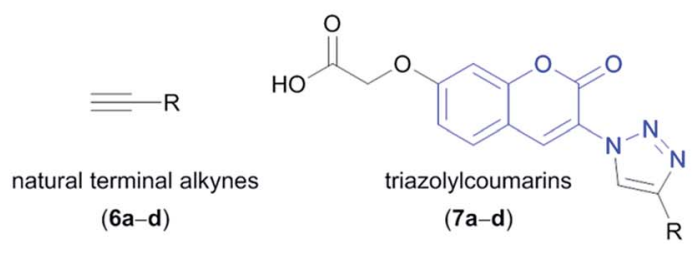

\begin{tabular}{|c|c|c|}
\hline & 6 & 7 \\
\hline
\end{tabular}
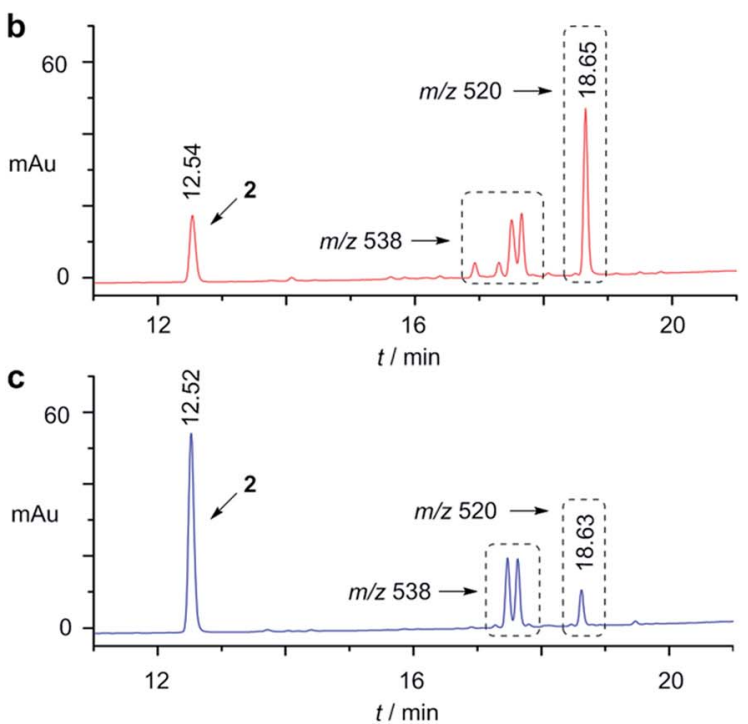

Fig. 4 Validation of the sensing system with the crude methanol extracts of $L$. japonica. (a) The chemical structures and molecular weights of the reported terminal alkyne-containing natural products $6 a-d$ in the leaves of L. japonica and the corresponding triazolylcoumarins 7a-d; (b) the LC chromatogram (at $345 \mathrm{~nm}$ ) of the off-bead sample from the methanol extract of the leaves of $L$. japonica; (c) the LC chromatogram (at $345 \mathrm{~nm}$ ) of the off-bead sample from the methanol extract of the stem heartwood of $L$. japonica.

observed in the LC/MS chromatogram in addition to the peak of 2 (Fig. 4b). The deduced molecular mass (521 Daltons) of the major peak (retention time: $18.65 \mathrm{~min}$ ) matched well with the theoretical molecular weight of $7 \mathbf{a}$, the triazolylcoumarin product resulting from the $\mathrm{CuAAC}$ reaction of $6 \mathbf{a}$. The other four peaks exhibited the same $m / z$ value of $538\left([\mathrm{M}-\mathrm{H}]^{-}\right)$. The 
deduced molecular masses (539 Daltons) are in good agreement with those of triazolylcoumarins $\mathbf{7 b}-\mathbf{d}$. These results suggested the presence of four terminal alkyne compounds $\mathbf{6 a - d}$ and one additional, as yet unidentified terminal alkyne compound with a molecular weight of 278 in the leaves of L. japonica, although we could not exclude the possibility that the stereoisomers of $\mathbf{6 a - d}$ are the structures of natural terminal alkynes.

The methanol extract of the stem heartwood of the same plant (3 mg) also activated the fluorescence of the beads. However, the cleaved products showed a somewhat different pattern of peaks in the LC/MS chromatogram compared to the leaf extracts, suggesting that the levels of specific terminal alkyne natural products 6a-d varied among different parts of the plant (Fig. 4c). Because of the characteristic and intense UV absorption of the triazolylcoumarin moiety, the quantification of individual terminal alkyne compounds was possible using the standard curve method. For example, on the basis of the respective integration values, compound $\mathbf{6 a}$ is estimated to exist in the methanol extract of the leaves at a concentration of approximately $1.0 \mathrm{mg} \mathrm{g}^{-1}$, whereas it is present at a concentration of approximately $0.2 \mathrm{mg} \mathrm{g}^{-1}$ in the extract of the stem heartwood (see ESI $\dagger$ ).

Encouraged by the successful results with the crude extract of $L$. japonica, we performed further investigations to isolate

a "Natural Product Extract Screening"

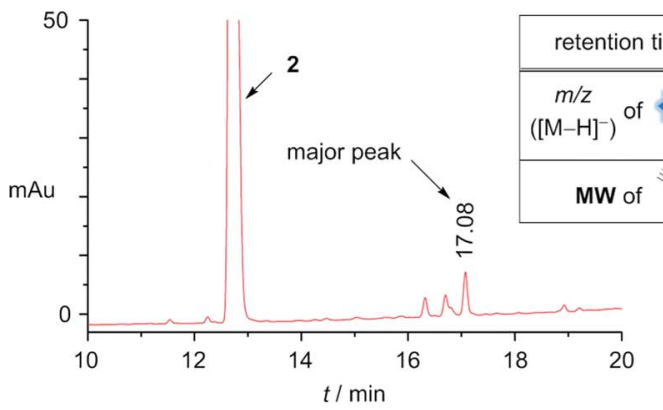
total volume $50 \mu \mathrm{L}$ sensing bead $1(0.5 \mathrm{mg})$ natural product extract (ca. $5 \mathrm{mg}$ )
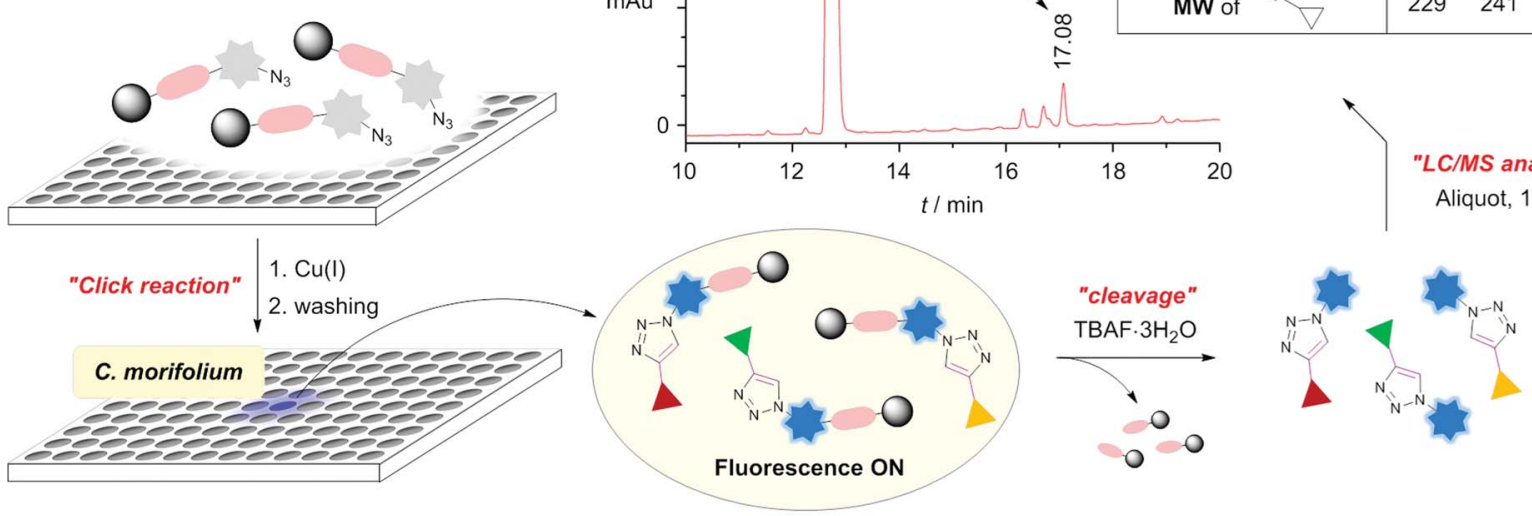

b "Chemical Assay-guided Isolation Process"

$=\mathrm{a}$ fraction that showed turn-on fluorescence response

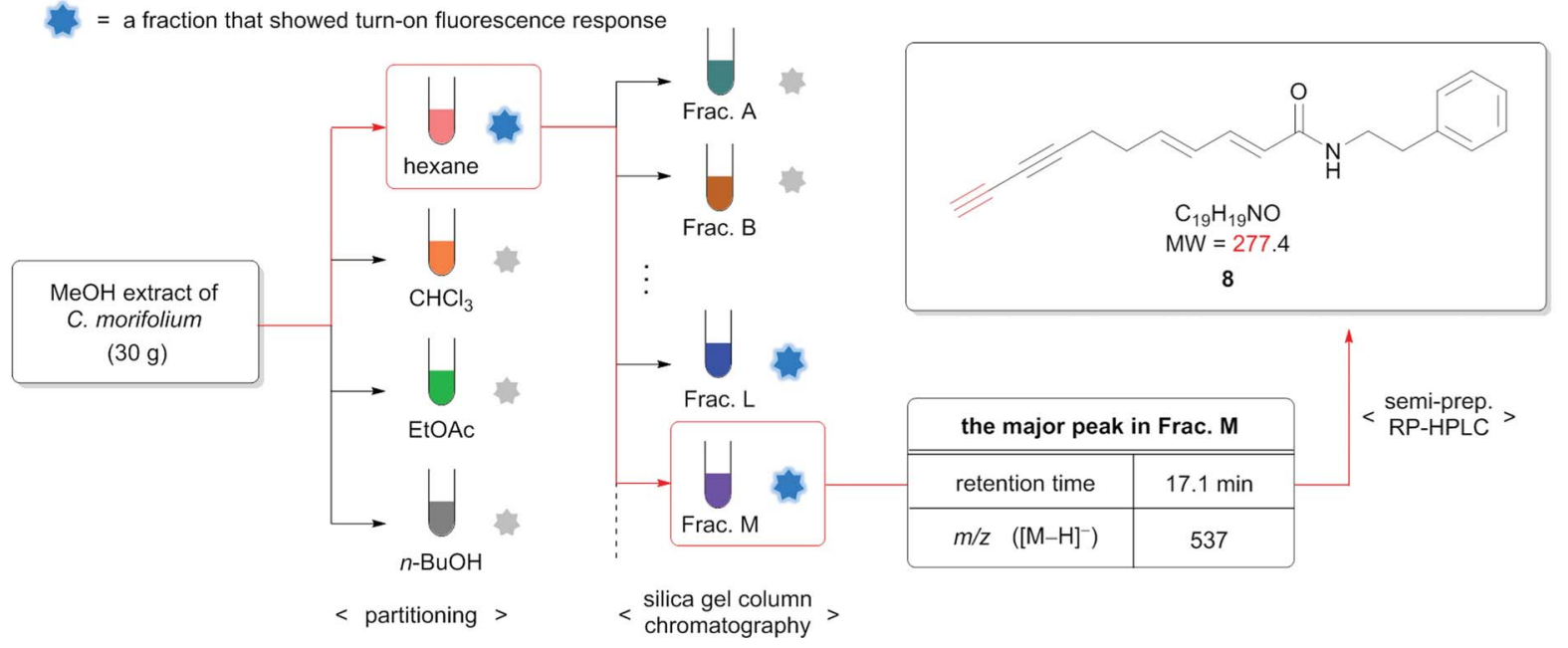

Fig. 5 Overall workflow of the natural product isolation process with alkyne sensing bead 1. (a) The various natural product extracts were screened in a multiwell plate with bead 1 for the presence of terminal alkynes. After the CuAAC reaction and a washing step, the beads that reacted with the methanol extract of the whole plant of $C$. morifolium exhibited fluorescence emission by fluorescence microscopy. The LC chromatogram at $345 \mathrm{~nm}$ of the reaction mixture released from the beads showed one major peak (retention time: 17.08 min) and two minor peaks (retention time: 16.32 and $16.70 \mathrm{~min}$ ). (b) To isolate the target natural compound, the specific fractions were selected by the click reaction with bead 1 and LC/MS. Subsequently, the selected fractions were subjected to purification steps by silica-gel column chromatography and semi-preparative RP-HPLC to give the pure terminal diyne 8 . Frac. = fraction. 
terminal alkyne compounds from the extracts of natural products using the alkyne sensing bead 1. Among the tested crude natural plant extracts of various origins, fluorescence activation was observed for the methanol extract of the whole plant of Chrysanthemum morifolium (Compositae) (Fig. 5a and ESI $\dagger$ ), from which no terminal alkyne compounds have previously been isolated.

Analysis of the LC/MS chromatogram after resin cleavage indicated the presence of three terminal alkyne compounds. To isolate the terminal alkyne component corresponding to the major peak, $30 \mathrm{~g}$ of the methanol extract was suspended in water and successively extracted with hexane, chloroform, ethyl acetate, and $n$-butanol (Fig. 5 b). A small portion of each fraction was subjected to the click reaction with alkyne sensing bead $\mathbf{1}$. Fluorescence activation was observed only in the hexane fraction. Fractionation of the hexane extract over silica gel produced fifteen different fractions (A-O). The chemical assay with bead 1 and LC/MS analysis indicated that the desired component was present primarily in fraction $\mathbf{M}$. Purification of fraction $\mathbf{M}$ by semi-preparative reverse-phase HPLC with the guidance of the extracted ion chromatograms afforded a pure compound with an $m / z$ value of $278[\mathrm{M}+\mathrm{H}]^{+}$as a white solid. Through analysis of the spectroscopic data, the isolated compound was determined to be terminal diyne $\mathbf{8 .}^{\mathbf{1 8}}$ The isolated compound is a previously reported natural product. ${ }^{19}$ However, this is the first report of the known natural compound $\mathbf{8}$ being isolated from the extract of C. morifolium.

\section{Conclusions}

In conclusion, we have developed a novel prototype chemical assay-guided natural product isolation method. Click chemistry was used in the chemical assay system to reliably identify target compounds without interference from other components in the extracts. A click reaction-induced fluorescence sensing platform was employed to sensitively detect even low concentrations of the target compounds and to permit visualization. Immobilization of this "sensing and reporting" moiety on a solid support was adopted for easy handling and tracking. The presented chemical assay system was designed for the detection of terminal alkyne-containing natural products using CuAAC, the prototypical click chemistry reaction. Our newly designed alkyne sensing bead $\mathbf{1}$ can successfully and quantitatively identify terminal alkyne compounds on the basis of a fluorescence signal. Using this bead, we were able to identify, quantify, and isolate terminal alkyne natural products from plant extracts, demonstrating the prospect of chemical assay-guided natural product isolation. Further studies, including the extension of this chemical assay system to other functional groups through the use of other viable click chemistry reactions, are needed to robustly establish chemical assay-guided natural product isolation. We believe that our "click reaction/fluorescent chemodosimetric probe/solid support" chemical assay system could also be applicable to numerous other fields, such as metabolomics and food science, which require the analysis of complex mixtures of compounds.

\section{Acknowledgements}

This work was supported by the Mid-Career Researcher Program (no. 2013R1A2A1A01015998) of the National Research Foundation of Korea (NRF) grant funded by the Korean government (MSIP). This work was also supported by BK21 Plus Program in 2014. We thank Prof. Won Keun Oh (Seoul National University) for helpful advice about natural product isolation.

\section{Notes and references}

1 For recent reviews, see: (a) A. L. Harvey, Drug Discovery Today, 2008, 13, 894-901; (b) J. W.-H. Li and J. C. Vederas, Science, 2009, 325, 161-165; (c) D. J. Newman and G. M. Cragg, J. Nat. Prod., 2012, 75, 311-335.

2 (a) A. M. Piggott and P. Karuso, Comb. Chem. High Throughput Screening, 2004, 7, 607-630; (b) E. E. Carlson, ACS Chem. Biol., 2010, 5, 639-653.

3 (a) L. Bohlin and J. G. Bruhn, Bioassay Methods in Natural Product Research and Drug Development, Kluwer Academic Press, Dordrecht, 1999; (b) A. A. Ströemstedt, J. Felth and L. Bohlin, Phytochem. Anal., 2014, 25, 13-28.

4 For recent reviews, see: (a) F. E. Koehn and G. T. Carter, Nat. Rev. Drug Discovery, 2005, 4, 206-220; (b) O. Sticher, Nat. Prod. Rep., 2008, 25, 517-554; (c) O. Potterat and M. Hamburger, Nat. Prod. Rep., 2013, 30, 546-564.

5 F. E. Koehn, in Natural Compounds as Drugs Volume I, ed. F. Petersen and R. Amstutz, Birkhäuser, Basel, 2008, vol. 65, pp. 175-210.

6 (a) E. E. Carlson and B. F. Cravatt, Nat. Methods, 2007, 5, 429435; (b) S. S. Ebada, R. A. Edrada, W. Lin and P. Proksch, Nat. Protoc., 2008, 3, 1820-1831.

7 (a) N. R. Farnsworth, J. Pharm. Sci., 1966, 55, 225-276; (b) S. Gibbons and A. I. Gray in Natural Products Isolation, ed. R. J. P. Cannell, Humana Press, New Jersey, 1988, vol. 4, ch. 4, pp. 209-245.

8 For reviews on click chemistry, see: (a) H. C. Kolb, M. G. Finn and K. B. Sharpless, Angew. Chem., Int. Ed., 2001, 40, 20042021; (b) H. C. Kolb and K. B. Sharpless, Drug Discovery Today, 2003, 8, 1128-1137.

9 (a) V. V. Rostovtsev, L. G. Green, V. V. Fokin and K. B. Sharpless, Angew. Chem., Int. Ed., 2002, 41, 25962599; (b) E. Lallana, R. Riguera and E. Fernandez-Megia, Angew. Chem., Int. Ed., 2011, 50, 8794-8804.

10 (a) A. L. K. Shi Shun and R. R. Tykwinski, Angew. Chem., Int. Ed., 2006, 45, 1034-1057; (b) V. Dembitsky and A. Siddiq, Anti-Cancer Agents Med. Chem., 2008, 8, 132-170; (c) D. V. Kuklev, A. J. Domb and V. M. Dembitsky, Phytomedicine, 2013, 20, 1145-1159.

11 For reviews on fluorescent chemodosimeters, see: $(a)$ D. G. Cho and J. L. Sessler, Chem. Soc. Rev., 2009, 38, 1647-1662; (b) C. Le Droumaguet, C. Wang and Q. Wang, Chem. Soc. Rev., 2010, 39, 1233-1239; (c) K. Kaur, R. Saini, A. Kumar, V. Luxami, N. Kaur, P. Singh and S. Kumar, Coord. Chem. Rev., 2012, 256, 1992-2028; (d) J. Chan, S. C. Dodani and C. J. Chang, Nat. Chem., 2012, 4, 973-984. 
12 M. Wagner and H. Kunz, Angew. Chem., Int. Ed., 2002, 41, 317-321.

13 K. Sivakumar, F. Xie, B. M. Cash, S. Long, H. N. Barnhill and Q. Wang, Org. Lett., 2004, 6, 4603-4606.

14 The product yield was calculated, after resin cleavage, according to the standard curve of triazolylcoumarin 3 (see ESI $\dagger$ ).

15 C. Shao, X. Wang, Q. Zhang, S. Luo, J. Zhao and Y. Hu, J. Org. Chem., 2011, 76, 6832-6836.

16 Y. Xia, W. Li, Z. Fan, X. Liu, C. Berro, E. Rauzy and L. Peng, Org. Biomol. Chem., 2007, 5, 1695-1701.

17 (a) H. Tanaka, T. Nakamura, K. Ichino, K. Ito and T. Tanaka, Phytochemistry, 1990, 29, 857-859; (b) B. S. Min, S. Y. Lee, J. H. Kim, O. K. Kwon, B. Y. Park, R. B. An, J. K. Lee,
H. I. Moon, T. J. Kim, Y. H. Kim, H. Joung and H. K. Lee, J. Nat. Prod., 2003, 66, 1388-1390.

18 For previous reports of trapping terminal polyynes by CuAAC, see: (a) T. Luu, R. McDonald and R. R. Tykwinski, Org. Lett., 2006, 8, 6035-6038; (b) C. Ross, K. Scherlach, F. Kloss and C. Hertweck, Angew. Chem., Int. Ed., 2014, 53, 7794-7798.

19 (a) R. Jente, P. H. Bonnet and F. Bohlmann, Chem. Ber., 1972, 105, 1694-1700; (b) R. Martin and H. Becker, Phytochemistry, 1985, 24, 2295-2300; (c) O. Hofer, H. Greger, W. Robien and A. Werner, Tetrahedron, 1986, 42, 2707-2716; (d) G. P. Li, B. C. Shen, J. F. Zhao, X. D. Yang and L. Li, J. Integr. Plant Biol., 2007, 49, 1608-1610; (e) V. Sharma, J. Boonen, B. D. Spiegeleer and V. K. Dixit, Phytother. Res., 2013, 27, 99-106. 